Peer Reviewed Paper openaccess

\title{
Increased sensitivity in near infrared hyperspectral imaging by enhanced background noise subtraction
}

\author{
Torbjørn Mehl, ${ }^{\text {a* }}$ Guro Marie Wyller, ${ }^{\mathrm{b}}$ Ingunn Burud ${ }^{\mathrm{c}}$ and Espen Olsen ${ }^{\mathrm{d}}$ \\ aDepartment of Mathematical Sciences and Technology, Norwegian University of Life Sciences, Universitetstunet 3, 1433 Ås, Norway. \\ E-mail: torbjorn.mehl@nmbu.no. (1) https://orcid.org/0000-0001-5766-9520 \\ ${ }^{b}$ Department of Mathematical Sciences and Technology, Norwegian University of Life Sciences, Universitetstunet 3, 1433 Ås, Norway, \\ Fraunhofer Institute for Solar Energy Systems ISE, Heidenhofstr. 2, 79110 Freiburg, Germany and Institute for Energy Technology IFE, \\ Instituttveien 18, 2007 Kjeller, Norway. 내 https://orcid.org/0000-0001-6452-3052 \\ 'Department of Mathematical Sciences and Technology, Norwegian University of Life Sciences, Universitetstunet 3, 1433 Ås, Norway. \\ 느 https://orcid.org/0000-0003-0637-4073 \\ dDepartment of Mathematical Sciences and Technology, Norwegian University of Life Sciences, Universitetstunet 3, 1433 Ås, Norway. \\ 느 https://orcid.org/0000-0001-9721-1637
}

\begin{abstract}
Near infrared hyperspectral photoluminescence imaging of crystalline silicon wafers can reveal new knowledge on the spatial distribution and the spectral response of radiative recombination active defects in the material. The hyperspectral camera applied for this imaging technique is subject to background shot noise as well as to oscillating background noise caused by temperature fluctuations in the camera chip. Standard background noise subtraction methods do not compensate for this oscillation. Many of the defects in silicon wafers lead to photoluminescence emissions with intensities that are one order of magnitude lower than the oscillation in the background noise level. These weak signals are therefore not detected. In this work, we demonstrate an enhanced background noise subtraction scheme that accounts for temporal oscillations as well as spatial differences in the background noise. The enhanced scheme drastically increases the sensitivity of the camera and hence allows for detection of weaker signals. Thus, it may be useful to implement the method in all hyperspectral imaging applications studying weak signals.
\end{abstract}

Keywords: near infrared, hyperspectral imaging, noise subtraction, photovoltaic, crystalline silicon

\section{Introduction}

The presence of defects and impurities in silicon wafers for photovoltaic (PV) applications leads to a significant reduction of the efficiency of the final solar cells. Until now, defects and impurities in solar cell wafers have been studied by microscopy such as scanning electron microscope $(S E M)^{1}$ and transmission electron microscopy $(\text { TEM })^{2}$ or scanning methods such as light beam induced current (LBIC). ${ }^{3}$ Recently electro- and photoluminescence
(EL and PL) have been developed as methods for lateral studies. ${ }^{4,5}$ Conventional EL and PL use non-spectrally resolved cameras which detect a broadband signal over a surface. Near infrared (NIR) hyperspectral imaging of photoluminescence from radiative recombination active defects is a method that can reveal new knowledge on both the spatial distribution and spectral response of these crystal imperfections. ${ }^{6}$ In the present work, a NIR

\section{Correspondence}

Torbjørn Mehl (torbjorn.mehl@nmbu.no)

Received: 11 October 2018

Revised: 28 December 2018

Accepted: 4 January 2019

Publication: 10 January 2019

doi: $10.1255 /$ jsi.2019.a2

ISSN: 2040-4565

\section{Citation}

T. Mehl, G.M. Wyller, I. Burud and E. Olsen, "Increased sensitivity in near infrared hyperspectral imaging by enhanced background noise subtraction", J. Spectral Imaging 8, a2 (2019). https://doi.org/10.1255/ jsi.2019.a2

(c) 2019 The Authors

This licence permits you to use, share, copy and redistribute the paper in any medium or any format provided that a full citation to the original paper in this journal is given. 
hyperspectral pushbroom camera is applied to study radiative defects in wafers and solar cells.

The hyperspectral image (HI) is an assembly of frames recorded over a timespan. Careful temperature control of the charge-coupled device (CCD) detector is needed in order to maintain a steady level of thermal noise. Variation in detector temperature may cause fluctuations in the noise level of the camera. The standard background noise subtraction method does not compensate for this. Normally this is not a problem, since the signals are significantly stronger than the background noise variation.

Many of the defects in silicon wafers lead-upon proper excitation-to PL emissions with intensities more than one order of magnitude smaller than the variation in the camera's background noise level. In this work, we demonstrate how it is possible to detect these weak signals by enhancing the background noise subtraction scheme of the camera. This will also be very relevant for other hyperspectral applications that study weak signals.

There are three primary sources of noise in a CCD image sensor: ${ }^{7}$ shot noise, read noise and dark noise. Shot noise (also called photon noise) is due to the inherent statistical variation in the arrival rate of photons incident on the CCD. The particle nature of light gives the incoming photons a Poisson distribution and the shot noise is thereby the square root of the signal. ${ }^{8}$ Read noise is from the digitisation process, where the on-chip preamplifier contributes most. Read noise is added uniformly to every pixel. In the CCD, at temperatures above OK, charge carriers will be excited thermally resulting in a thermally dependent current causing a noise signal. The statistical variation of this electron generation is called dark noise, has a Poisson distribution, and is the square root of electrons generated during exposure time.

In addition, there can be a background photon flux incident on the CCD due to scattered light from the surroundings. Even with the shutter closed, black body radiation from inside the camera, e.g. the shutter itself, may be recorded due to temperatures above OK in the apparatus.

The signal-to-noise ratio can then be described by Equation (1).

$$
S N R=\frac{P Q_{e} t}{\sqrt{(P+B) Q_{e} t+D t+N_{r}^{2}}}
$$

$P$ is the photon flux from sample, $Q_{e}$ is the quantum efficiency of the CCD, $t$ is the exposure time, $B$ is the background photon flux, $D$ is the dark current and $N_{r}$ is the read noise. The sum of these is what is referred to as the background noise in this study.

Impulse noise is mainly caused by dead pixels, analogto-digital converter errors and bit errors in transmission, and is often a temporary disturbance. It can appear as salt-and-pepper grains in a 2-D grey scale digital image and is often removed by general pixel cleaning, i.e. spatial and/or spectral median based filters. ${ }^{9}$ Used on a 3-D HI it may compromise some of the spectral and/or spatial data. In a HI the impulse noise can be observed as stripes in the scanning direction, $y$, after the dark-frame subtraction. Since most of the impulse noise is of temporary nature, a stripe will only appear (partly) in one of subsequent recorded images.

To remove most of the impulse noise without compromising the data, the median of corresponding values from three subsequently recorded HIs is used. In this process, a time dependent additive dark level was detected in the frames. The present work demonstrates how these variations can be corrected.

\section{Materials and method}

$\mathrm{PL}$ imaging is a standard technique used for solar cell materials where photo-excitated charge carriers emit photons when they relax to the valence band. As excitation source, the hyperspectral PL setup uses an $808 \mathrm{~nm}$ line laser with an irradiated power density of $2 \mathrm{~W} \mathrm{~cm}^{-2}$ (Lasiris Magnum II). An $850 \mathrm{~nm}$ long-pass filter prevents laser beam reflections from entering the optics.

A NIR hyperspectral camera (SWIR, Specim, Finland), with an $\mathrm{HgCdTe}$ detector with a spectral resolution of $6 \mathrm{~nm}$ in the range $929-2531 \mathrm{~nm}(1.33-0.49 \mathrm{eV})$ was used. The camera records a 2-D frame with one 320-pixel spatial dimension $(x)$ and one 256-band spectral dimension ( $\lambda$ ). The resolution of the measured value for a sensor is 14 bits, that is, values in the range of 0 to 16,383 and referred to as counts. A count is proportional to the quantity of photons detected by the sensor. By assembling frames taken while the camera scans the sample, the second spatial dimension (y) is generated. This setup yields a three-dimensional data file (spatial $x, y$ and spectral $\lambda$ ), called a hypercube. This provides a PL spectrum in each spatial pixel $(x, y)$ of the sample. Images were taken of samples cooled with liquid nitrogen to $90 \mathrm{~K}$. A similar setup has been used 


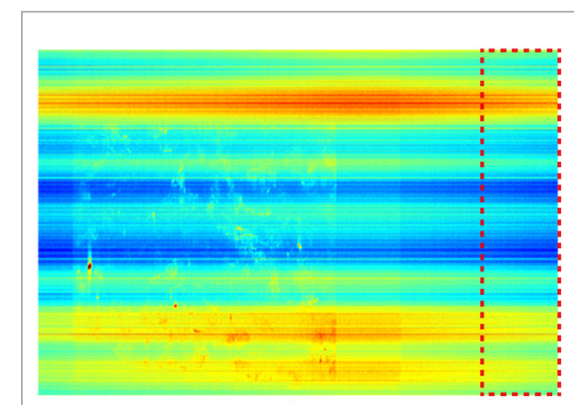

(a)

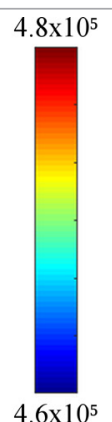

$4.6 \times 10^{5}$

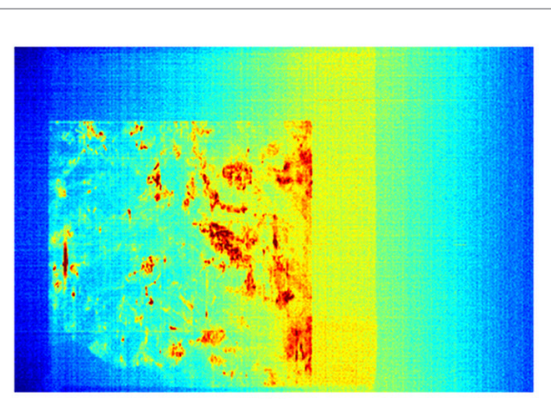

(b)

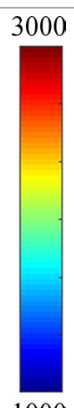

$-1000$

Figure 1. Image (a) is an integral over defect-related PL in the range $0.73-0.92 \mathrm{eV}$ (wavelength bands 67-124) before, whereas image (b) is after, standard dark-frame background noise subtraction. The dashed rectangle in image (a) shows the "dark area" from which the background noise level for each pixel is found. After standard subtraction scheme, a gradient can be seen in the scanning direction (horizontal) of image (b).

in previous studies. $6,10,11$ The scanning speed for $\mathrm{PL}$ mapping was approximately $13 \mathrm{~mm} \mathrm{~s}^{-1}$.

The sample holder used allows for sample sizes up to $156 \times 156 \mathrm{~mm}^{2}$. The sample used in this study is a $125 \times 125 \mathrm{~mm}^{2}$ multi-crystalline silicon wafer. There is thus a 60-pixel wide area outside the edges of the sample where the sample holder's surface is exposed, as seen in Figure 1b. The small sample size was chosen for a better illustration of the variation in background noise because the exposed sample holder surface functions as a homogeneous reference area.

Since PL from defects in wafers and solar cells are weak signals, images recorded are performed with an exposure time of $20 \mathrm{~ms}$. This leads to a relatively high background noise level. The background noise level is around 9000 counts, while the PL signal is from a few tens to several thousand counts. As shown in Figure 1, the background noise level is more than one order of magnitude larger than the defect-related PL signal. Moreover, the background noise level varies from pixel to pixel.

\section{Standard background noise subtraction method}

The standard method to remove background noise is to subtract a dark frame, recorded with the shutter closed, from the unprocessed raw frame, recorded with shutter open. The dark frame is recorded by keeping the shutter closed either during the first and/or last part of the recording, depending on the instrument and settings. In our setup, we keep the shutter closed for the last three seconds. This results in a dark area at the end of the picture exhibiting no luminescence signal (dashed rectangle in Figure 1a). The background noise level value for each pixel of the camera chip is found as the median value from several successive frames in the dark area. Here we have used 50 successive frames. In the $x, y$, $\lambda$-coordinate system, this means that the background noise for pixel $\left(x_{i}, \lambda_{k}\right)$ on the two-dimensional camera chip is found by taking the median of pixel values from $\left(x_{i}, y_{j}\right.$, $\left.\lambda_{k}\right)$ to $\left(x_{i}, y_{j+50}, \lambda_{k}\right)$ in the hypercube. The resulting matrix is referred to as the background noise matrix (BNM), which is our dark frame.

\section{Differences between subsequent measurements}

Figure $1 \mathrm{~b}$ shows a gradient in the image after the standard background noise subtraction (scanning from left to right in the horizontal direction). A comparison of three subsequent measurements of the same wafer, with equal measurement parameters, is shown in Figure 2. The count rate seems to increase from left to right during the measurements (a) and (c), whereas it decreases from left to right during measurement (b). This feature suggests that the background noise level fluctuates periodically and regardless of recording start time.

\section{Identification of oscillations in background noise level}

Based on Figure 2, it was suspected that the difference in count rate was caused by fluctuations in the background noise level of the camera detector during the measurements. In order to investigate this, measurements were conducted over a long time-interval (200s) with a closed shutter, thus allowing only the background noise level to be recorded. A plot of the count rate for each pixel $\left(x_{1}\right.$, $\lambda_{j}$ ) for one spatial value, $x_{1}$, along the central part of the 


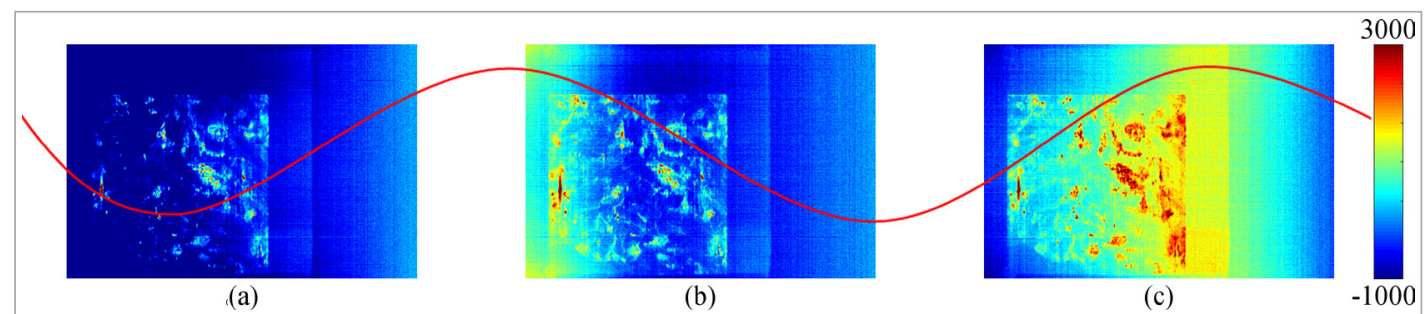

Figure 2. Defect-related PL signal from three subsequent measurements; (a), (b) and (c). During measurements (a) and (c), the count rate increases, whereas it decreases during measurement (b). This feature suggests some variable is fluctuating periodically, indicated by a red line.

camera chip as a function of time (Figure 3), confirmed our suspicion of a periodically oscillating behaviour of the background noise. Figure 3 illustrates both the difference in background noise level from pixel to pixel, and the oscillation of the background noise level for all pixels as a function of time. The oscillation is believed to be caused by the CCD chip temperature control algorithm. The oscillation fits an on/off cooling regime with period, $T \approx 45 \mathrm{~s}$.

With cooling turned off, the temperature will rise, causing the dark noise to increase. When the temperature reaches a certain level, the cooling is activated, causing the temperature, and thus the dark noise level, to decrease. After reaching a certain minimum temperature, the cooling system would again be deactivated.

A hyperspectral PL image with spatial resolution of $500 \mu \mathrm{m}$ will take approximately $18 \mathrm{~s}$ to record when scanning a full size $156 \times 156 \mathrm{~mm}^{2}$ wafer/solar cell. For higher resolution, the recording time increases, e.g. applying a macro lens with a resolution of $27 \mu \mathrm{m}$, a recording takes more than four minutes. This means that the oscillation will have an impact on the results, especially when the signal intensities are in the same order of magnitude, or lower, than the amplitude of the oscillation.

\section{Spatial variation of background noise level on camera chip}

The next step was to investigate whether the oscillation in background noise level has the same amplitude for every location on the camera chip. To investigate the pixel-wise maximum and minimum of the oscillation, a recording with the shutter closed for more than one period was taken. Based on the difference between the minimum and the maximum value, a matrix with the same dimensions as the camera chip was constructed. For each camera pixel $\left(x_{i}, \lambda_{j}\right)$, the corresponding pixel in the matrix ( $i, j)$ gives the count difference between the highest and lowest possible background noise level. In the following, this matrix is referred to as the background amplitude correction matrix (BACM).

We discovered that the background oscillation amplitude varies over the chip surface. The highest amplitude

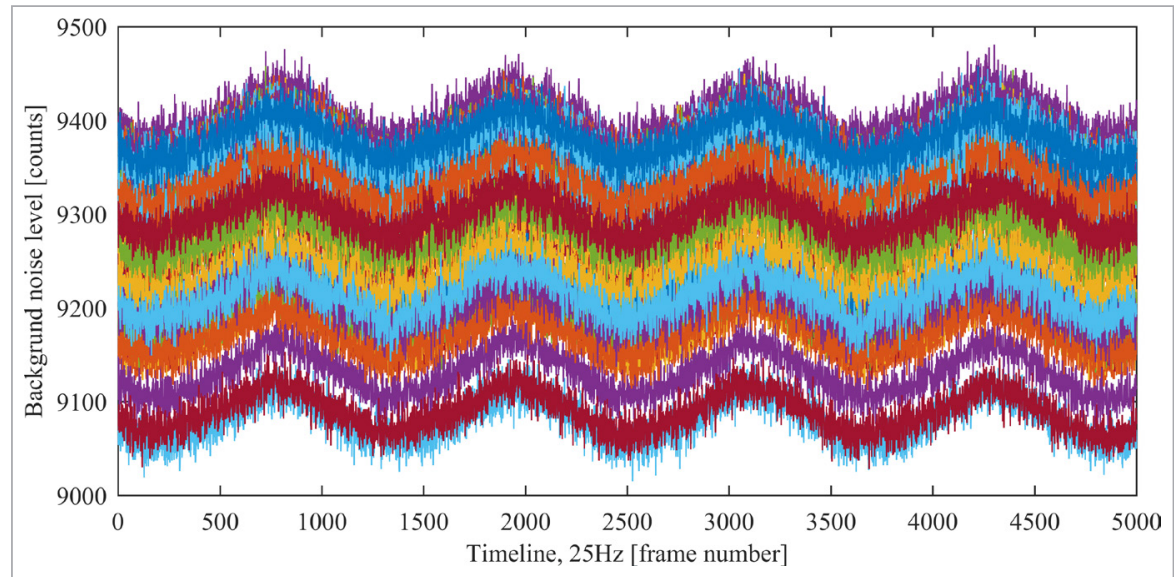

Figure 3. Oscillations in background noise level. All sensors oscillate with the same period, $T \approx 45 \mathrm{~s}$ and are in phase. 


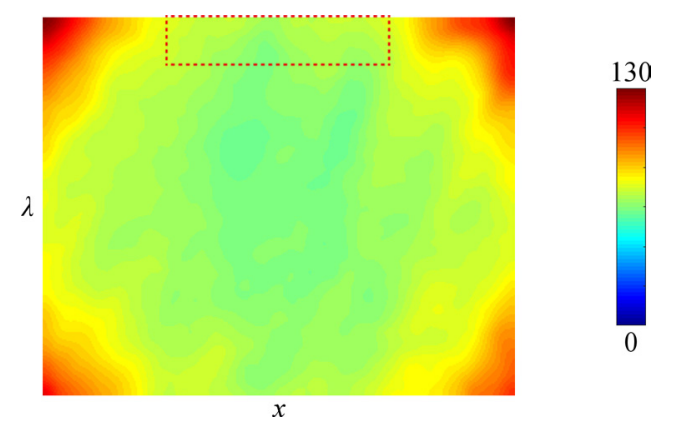

Figure 4. Visualisation of the background amplitude correction matrix (BACM). Each pixel gives the difference between the highest and the lowest background noise level for the corresponding point on the camera chip. The dashed rectangle indicates the reference area of the matrix (see text). The median value for this area is referred to as the background amplitude correction matrix reference (BACMref).

was found in the corners of the chip (120 counts). In the central area, however, 60 counts were recorded. A visualisation of the BACM is seen in Figure 4. From the picture it appears that the heat transfer is highest from the corners of the chip. This may be due to how the chip is mounted.

\section{Development of procedure to correct for oscillation}

Given that the oscillation in the background noise level has a period of $45 \mathrm{~s}$ and the hyperspectral $\mathrm{PL}$ recording time often is as short as $18 \mathrm{~s}$, it is not possible to determine where in the period the recording takes place. It was, therefore, decided to use another property of the oscillation. Since the amplitude of the oscillations is known and stored in the BACM, it is possible to determine the fraction of the amplitude between two frames in the recording, e.g. between the first and last (the dark) frame. This fraction can be used to compensate for the oscillation.

For this purpose, it is desirable to find an area on the chip that does not record sample-related signals during the entire measurement. It is also possible to use either a white reference or an area of the recording, spatial and/ or spectral, where the incoming signal is constant during measurement. Because the variation of counts with time in such an area would only be caused by the oscillation in the background noise level, this area could function as an amplitude reference. The dashed rectangle in Figure 4 indicates such a reference area of the matrix. The median of the values in this area is referred to as the background amplitude correction matrix reference (BACMref). Note that both the matrix BACM and the number BACMref are assumed to be constant; they depend neither on the frame number nor on the sample to be studied. It is to be noted that BACM depends on exposure time, cf. Equation (1).

\section{Working principle of the enhanced scheme}

Based on the reference area and the BACM, an enhanced background noise subtraction scheme is developed. For each raw frame, $\mathrm{RF}(\mathrm{y})$, the median number of counts in the reference area is calculated and stored in the raw frame reference, $\operatorname{RFref}(\mathrm{y})$. The background noise matrix reference, BNMref, of the measurement is found as the median number of counts in the reference area of the BNM. The delta thermal background, $\triangle \mathrm{TB}(\mathrm{y})$, is defined as the difference between a frame's background noise and the background noise matrix. $\triangle \mathrm{TB}(\mathrm{y})$ holds the extra counts that, due to thermal oscillations, need to be subtracted from each pixel in each raw frame, in addition to the BNM.

In the development of the new scheme we make the following two assumptions. First, we assume that the ratio of the thermal background $\triangle T B$ to the BACM (which is the maximum value of the thermal background oscillation) is equal over the entire camera chip for each frame, expressed in Equation (2).

$$
\frac{\Delta \operatorname{TB}\left(x_{1}, y_{1}, \lambda_{1}\right)}{\operatorname{BACM}\left(x_{1}, \lambda_{1}\right)}=\frac{\Delta \operatorname{TB}\left(x_{2}, y_{1}, \lambda_{2}\right)}{\operatorname{BACM}\left(x_{2}, \lambda_{2}\right)}=\text { constant }
$$

Then we assume that it will also hold for the median values from the reference area.

$$
\frac{\Delta \operatorname{TB}\left(x_{i}, y_{j}, \lambda_{k}\right)}{\operatorname{BACM}\left(x_{i}, \lambda_{k}\right)}=\frac{\operatorname{RFref}\left(y_{j}\right)-\text { BNMref }}{\text { BACMref }}
$$

This gives

$$
\Delta \mathrm{TB}(y)=\frac{\operatorname{RFref}(y)-\text { BNMref }}{\text { BACMref }} \cdot \text { BACM }
$$

The standard dark-frame subtraction scheme can be written as

$$
\mathrm{RF}(\mathrm{y})-\mathrm{BNM}=\mathrm{CF}(\mathrm{y})
$$

where CF is the corrected frame. To enhance the background correction scheme, by including subtraction of the extra counts due to thermal oscillations, $\triangle \mathrm{TB}(\mathrm{y})$, Equation (5) is modified by inserting Equation (4). CF is then defined as 


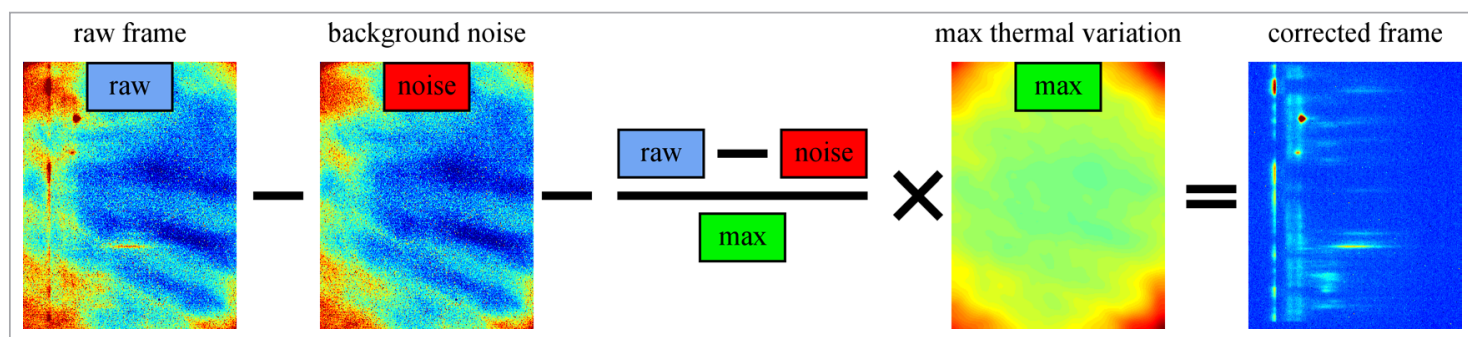

Figure 5. The enhanced subtraction regime; a visualisation of Equation (6). The background noise and the extra counts, due to thermal oscillation, are subtracted. The red, blue and green rectangles symbolise three constants, found from the median of the values from the reference area. The extra counts are calculated using the deviation in the reference area divided by maximum deviation.

$$
\mathrm{RF}(y)-\mathrm{BNM}-\frac{\mathrm{RFref}(\mathrm{y})-\mathrm{BNMref}}{\mathrm{BACMref}} \bullet \mathrm{BACM}=\mathrm{CF}(\mathrm{y})
$$

Equation (6) holds the enhanced scheme and is shown in Figure 5.

\section{Results and discussion}

\section{Determination of a reference area}

When measuring silicon wafers, the reference area can either be taken from the part of the chip which detects light with energy higher than the bandgap of silicon $(1.1 \mathrm{eV})$, or from the part detecting light with energy lower than the defect signals. Further investigation of photon energies $>1.25 \mathrm{eV}$, bands $1-10$, as a function of frame number, shows that there are some weak signals (Figure 6b). Thus, these bands cannot be used as a reference area. For bands 237-256, corresponding to photon energies $<0.52 \mathrm{eV}$, we conclude that there is no signal from the sample, see Figure $6 c$. These bands can thus be used as a reference area. The shutter is closing at frame 390 and a small increase in counts is observed. This is presumably black body radiation from the shutter itself.

Since the values in the corners of BACM differs from the middle part of the matrix, only $x$-values from 70 to 250 were used as reference area, as seen in Figure 4.

\section{Comparison of images after enhanced background correction}

To evaluate the new subtraction scheme, we made a comparison with the old, using the three subsequent measurements shown in Figure 2. By looking at weak $0.70 \mathrm{eV}$ PL signals from defects detected by band 136 , the mean number of counts for all $x$-values as a function of frame number is calculated from both the standard and the new subtraction regime of three measurements, see Figure 7 .

The possibility to detect and study weak signals in the measurements has increased drastically. The periodic oscillation is removed and the signal from the sample
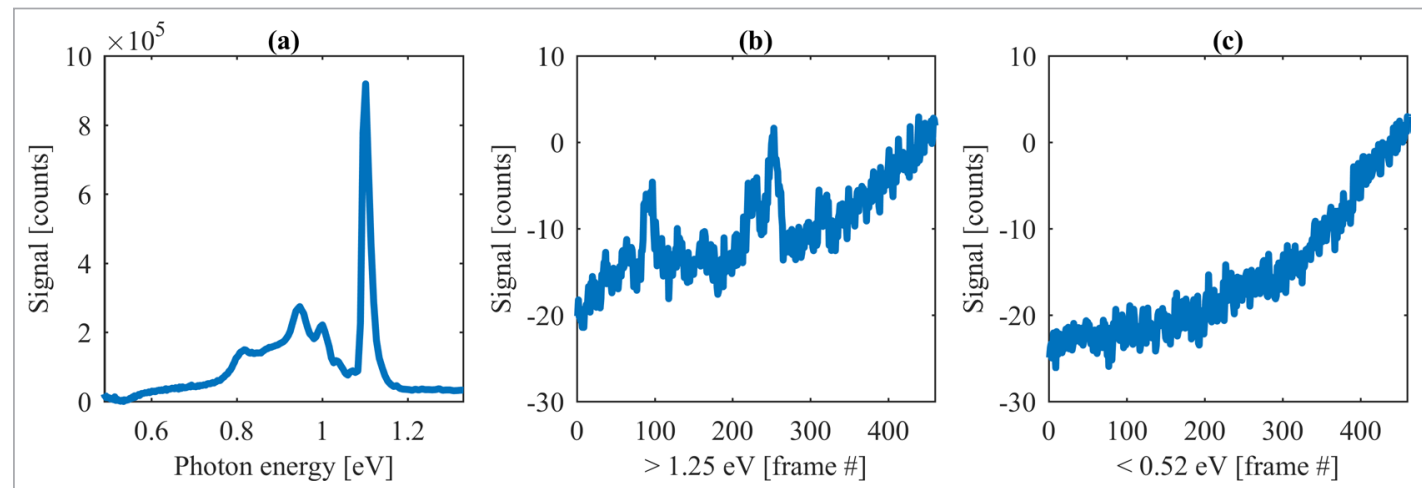

Figure 6. (a) Total spectrum from a hyperspectral PL measurement of a multicrystalline-Si wafer. (b) Mean number of counts for wavelength bands $1-10$, photon energies $>1.25 \mathrm{eV}$ and (c) for bands 237-256, photon energies $<0.52 \mathrm{eV}$, as function of frame number. Note that the frame numbers can be seen as a time dimension. Bands 237-256 receive no signal, only thermal oscillation. Thus, this part is used as reference area. 

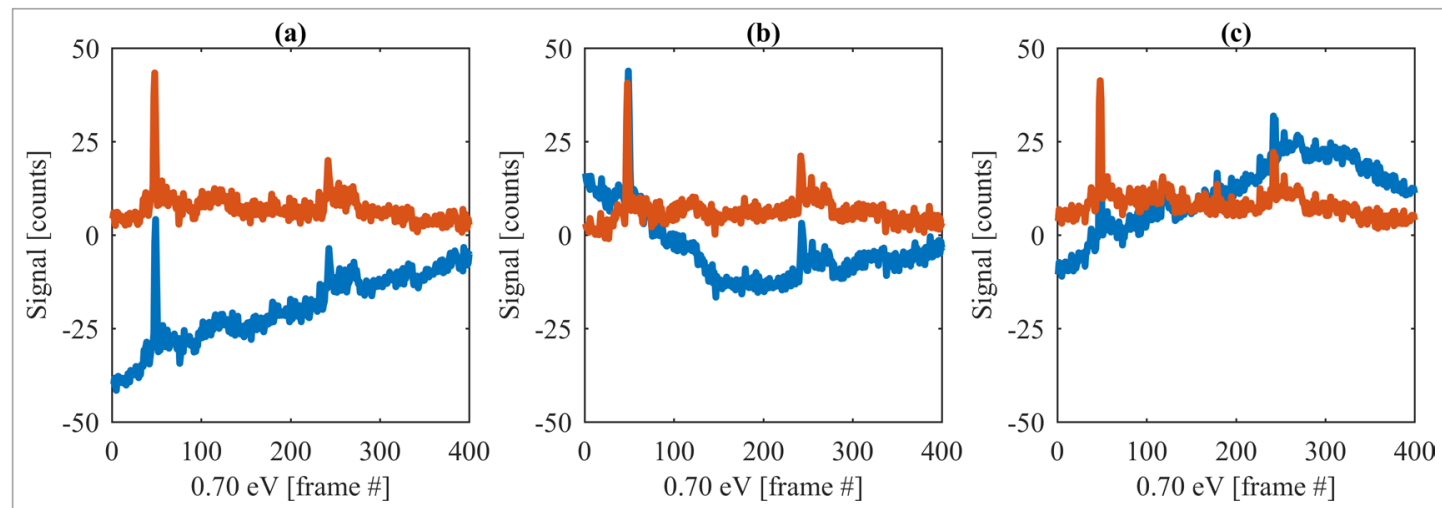

Figure 7. Weak PL signals from defects detected by band $136(0.70 \mathrm{eV})$. Mean number of counts for all $x$-values as a function of frame number, shown with both standard (blue) and new (red) subtraction regime of three subsequent measurements of the same sample; (a), (b) and (c). With the new subtraction regime, the measurements are comparable.

is more reproducible (Figure 7). This indicates that the assumption made in the description of the background correction procedure above seems to hold true.

Images of the three measurements (Figure 2) corrected with the new regime are shown in Figure $8 a-c$. Before the correction, the periodic oscillation, with an amplitude of 60-120 counts, limited the detection. With the new regime, the periodic signal is removed and the detection limit is set only by the standard deviation of the shot noise, which in this case is 14 counts.

\section{Median of three subsequent recordings}

With the periodic background noise removed, the possibility to remove most of the impulse noise, using the median of the corresponding values from subsequent recorded images, is enhanced. An image of the median of the three recordings is shown in Figure $8 \mathrm{~d}$. In addition, the detection limit is enhanced. The standard deviation in the background noise is reduced to six counts in the median image.

\section{Conclusion}

In summary, a thermal oscillation causing temporal variation in the background noise level of the hyperspectral camera is identified. An enhanced background noise subtraction scheme to correct for this has been devel-

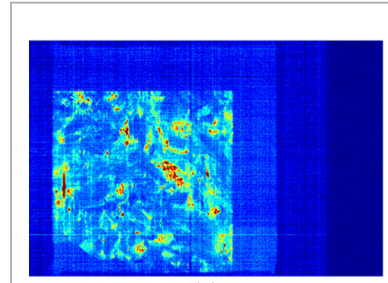

(a)

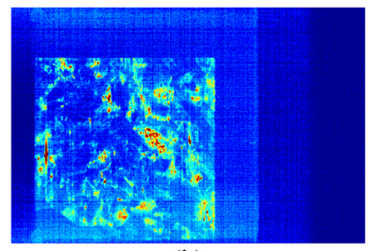

(b)

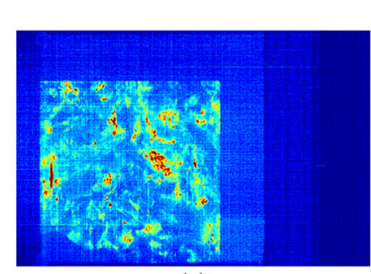

(c)
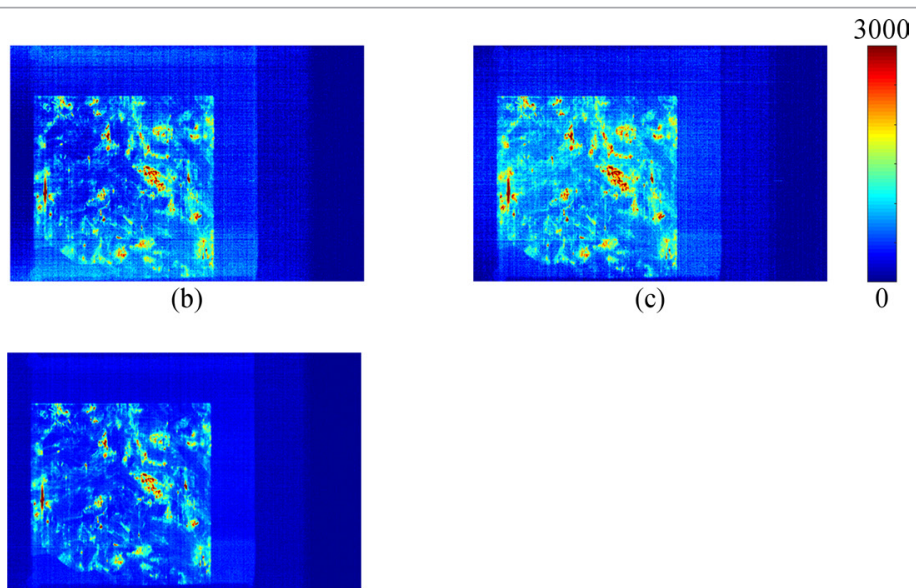

(d) 
oped. The PL signals from defects in the studied silicon wafers range from tens to several thousand counts. Taking into account that the amplitude of the background oscillation can attain a value of more than a hundred counts, defects with the weakest signals might not be detected. With the enhanced background subtraction scheme, the camera's sensitivity is drastically increased, allowing even these weakly emitting defects to be detected.

The enhanced background subtraction scheme may be used in all hyperspectral imaging applications. This scheme needs either a white reference in the scanning direction, or that some part of the incoming signals spectrum is constant or zero during the measurement. In particular, it may be useful to implement the method in applications studying weak signals.

\section{Acknowledgement}

This study has been a part of the FME-SuSolTech centre for renewable energy technology sponsored by the Norwegian Research Council together with Norwegian industrial partners. Support from the European Commission through the Seventh Framework Program project "SOPHIA-Solar Photovoltaic European Research Infrastructure" is greatly acknowledged (Grant 262533).

\section{References}

1. V. Higgs and M. Kittler, "Investigation of the recombination activity of misfit dislocations in $\mathrm{Si} /$ SiGe epilayers by cathodoluminescence imaging and the electron-beam-induced current technique", Appl. Phys. Lett. 63, 2085 (1993). https://doi. org/10.1063/1.110600

2. S. Binetti, J. Libal, M. Acciarri, M. Di Sabatino, H. Nordmark, E.J. Ovrelid, J.C. Walmsley and R. Holmestad, "Study of defects and impurities in multicrystalline silicon grown from metallurgical silicon feedstock", Mater. Sci. Eng. B.-Adv 159-60, 274 (2009). https://doi.org/10.1016/j.mseb.2008.05.013
3. B. Moralejo, M.A. González, J. Jiménez, V. Parra, O. Martínez, J. Gutiérrez and O. Charro, "LBIC and reflectance mapping of multicrystalline Si solar cells", J. Electron. Mater. 39, 663 (2010). https://doi. org/10.1007/s11664-010-1174-8

4. D. Mankovics, A. Klossek, C. Krause, T. Arguirov, W. Seifert and M. Kittler, "Luminescence of defects and breakdown sites in multicrystalline silicon solar cells", Phys. Status Solidi A 209, 1908 (2012). https:// doi.org/10.1002/pssa.201200133

5. T. Mchedlidze, W. Seifert, M. Kittler, A.T. Blumenau, B. Birkmann, T. Mono and M. Muller, "Capability of photoluminescence for characterization of multicrystalline silicon", J. Appl. Phys. 111, 073504 (2012). https://doi.org/10.1063/1.3699275

6. E. Olsen and A.S. Flø, "Spectral and spatially resolved imaging of photoluminescence in multicrystalline silicon wafers", Appl. Phys. Lett. 99, 3 (2011). https://doi.org/10.1063/1.3607307

7. T.J. Fellers and M.W. Davidson, CCD Noise Sources and Signal-to-Noise Ratio. Olympus America, Inc. and The Florida State University (2015). Available at: https://micro.magnet.fsu.edu/primer/digitalimaging/ concepts/ccdsnr.html [accessed: 5 April 2018].

8. S.W. Hasinoff, "Photon, Poisson noise", in Computer Vision, Ed by K. Ikeuchi. Springer, Boston, MA (2014). https://doi.org/10.1007/978-0-387-31439-6_482

9. S.H. Teoh and H. Ibrahim, "Median filtering frameworks for reducing impulse noise from grayscale digital images: a literature survey", Int. J. Fut. Comput. Commun. 1, 323 (2012). doi: https://doi. org/10.7763/IJFCC.2012.V1.87

10. D. Lausch, T. Mehl, K. Petter, A.S. Flø, I. Burud and E. Olsen, "Classification of crystal defects in multicrystalline silicon solar cells and wafer using spectrally and spatially resolved photoluminescence", J. Appl. Phys. 119, (2016). https://doi. org/10.1063/1.4940711

11. I. Burud, T. Mehl, A. Flo, D. Lausch and E. Olsen, "Hyperspectral photoluminescence imaging of defects in solar cells", J. Spectral Imaging 5, a8 (2016). https://doi.org/10.1255/jsi.2016.a8 